\title{
Using Sidereal Rotation Period Expressions to Calculate the Sun's Rotation Period through Observation of Sunspots
}

\author{
Tsung-Ching Chen, ${ }^{1}$ Jiann-Shing Lih, ${ }^{1}$ Tien-Tang Chang, ${ }^{2}$ Chih-Hung Yang, \\ Ming-Chi Lu, ${ }^{1}$ Chia-Ju Liu, ${ }^{2}$ and Ming-Chung Ho ${ }^{1}$ \\ ${ }^{1}$ Department of Physics, National Kaohsiung Normal University, Kaohsiung 824, Taiwan \\ ${ }^{2}$ Graduate Institute of Science Education \& Environmental Education, National Kaohsiung Normal University, Kaohsiung 824, Taiwan
}

Correspondence should be addressed to Ming-Chung Ho; t1603@nknucc.nknu.edu.tw

Received 30 September 2014; Accepted 26 December 2014

Academic Editor: Mo Li

Copyright (C) 2015 Tsung-Ching Chen et al. This is an open access article distributed under the Creative Commons Attribution License, which permits unrestricted use, distribution, and reproduction in any medium, provided the original work is properly cited.

\begin{abstract}
We utilize sidereal rotation period expressions to calculate the sun's rotation period via sunspot observation. From the well-known astronomical sites, we collected sunspot diagrams for 14 months, from January 2013 to February 2014, to analyze, compare, and implement statistical research. In addition to acquiring the average angular rate of the movement of sunspots, we found that even the same number of sunspots moved at different angular rates, and generally the life of larger sunspots is longer than 10 days. Therefore the larger sunspots moved around the back of the sun, and a handful of relatively smaller sunspots disappeared within a few days. The results show that the solar rotation period varied with the latitude. However, if we take the average of the sunspots at high and low latitudes, we find that the calculated value is very close to the accredited values.
\end{abstract}

\section{Introduction}

Astronomy observation has long been of interest. In the universe, our own solar system is the easiest system for us to make astronomical observations. In the solar system, the sun plays the major role. In many of the previous studies on solar observation, it is said that the sun's rotation period is approximately 27 days; however, how is this value obtained? Since the sun revolves, is the axis of rotation of the sun sloping as is that of the Earth? From the view of observers on Earth, does the rotation period of the sun differ with the four seasons of the year? These are questions that researchers are highly interested in. Previous studies have only shown that the solar rotation period can be estimated by the method of recording sunspots according to their projections. However, professional training is required to use quickly and accurately; otherwise there will be great artificial errors. Therefore, in our study, we propose another scheme to acquire the rotation period of the sun; that is, we make use of the well-known website of the World Data Center for the production, preservation, and dissemination of international sunspot numbers which records astronomical observations of sunspots, http://www.sidc.be/sunspot-data/, to statistically analyze the reasons for the trajectory and direction of the movement of sunspots on the solar disk. Such data can be used to identify changes in the angles of the sun's rotation axis. By observing the moving angular rates of sunspots on the solar disk and combining these rates with sidereal rotation period expressions to calculate the solar rotation period, the results show that we can not only estimate the solar rotation period more conveniently and accurately, but also further simulate the movement trajectory of sunspots and estimate the change and the sloping angle of the sun's rotation axis relative to that of the Earth. This paper is organized as follows: Section 2 provides a review of the topic, Section 3 presents the method, the results and discussion are described in Section 4, and, finally, Section 5 presents the conclusions.

\section{Review}

2.1. Sunspot Cycles. The phenomenon easiest to be observed on the surface of the sun is that of sunspots. We can use 
a variety of observation methods such as projection and solar filters to make observations of sunspots. When sunspots appear, they move over the surface of the sun, and from observations of this movement it is possible to calculate the sun's rotation period. In 1610, based on his observations of sunspots, Galileo found that the sun's rotation period is approximately 27 days. There has been an increase in the number of sunspots and a reduction in cyclical changes, a fact which was first found by the astronomer H. Schwabe in 1843. The number of sunspots has a cycle of approximately 11.1 years, which is known as the Schwabe cycle, after H. Schwabe's observations. In addition, the Babcock model places out of the magnetic field and is involved to explain this cycle [1]. The sun's surface is the most active when there is increased sunspot activity, while when the sunspot number is almost close to zero, it is referred to as the Maunder minimum.

Research "campaign period" of different latitude sunspot, you can find solar differential rotation phenomenon [2]; that is, there are differences in the rotation period at different latitudes. The sun's rotation period is 25 days at the equator, while at the extreme zones it increases to 35 days. Christopher Scheiner was the first to measure the rotation speed of the solar equator. He also noted that the rotation of the sun at high latitudes is slower than at the equator, and it is also possible that he was the discoverer of the sun's rotation differences [3].

2.1.1. Significance of Large Sunspot Groups. Sunspots are phenomena whereby strong and confused solar surface magnetic fields, in a relatively low temperature region, cause easy solar storms to erupt (these storms include solar flares, eruptive prominence, and coronal mass ejections). Thus, the number of sunspots represents the strength of the sun's surface activities.

The largest sunspot group is more than 13 times the Earth's surface. Solar flares and coronal mass ejections speed into space profusion of charged particles known as a plasma cloud. When this stream of charged particles crosses near the Earth, it will cause disturbances in the Earth's electromagnetic field, which may then lead to disruptions to telecommunications.

In high latitudes, the phenomenon of beautiful auroras or "magnetic storms" occurs. These "storms" may cause damage to communication satellites [4], power generation facilities, and other faults, affecting satellite television and radio communications, GPS, mobile phones, and flight facilities. Life on Earth is protected by the Earth's magnetic field and atmosphere, so it will not be directly harmed by these solar storms. However, astronauts in space stations are at risk because of their lack of protection, as they are directly exposed to intense radiation, from the sun. Therefore, if there is a large complex sunspot group in the sun facing the Earth, experts will repeatedly warn that all telecommunications, communications, air traffic, and power generation units must pay attention to the development of the situation.

The sun is the most important source of energy for the Earth. Its activity has a far-reaching impact on the state of the Earth's ecology and environment. Therefore, any movement on the surface of the sun is important information for scientists to investigate in relation to environmental changes.

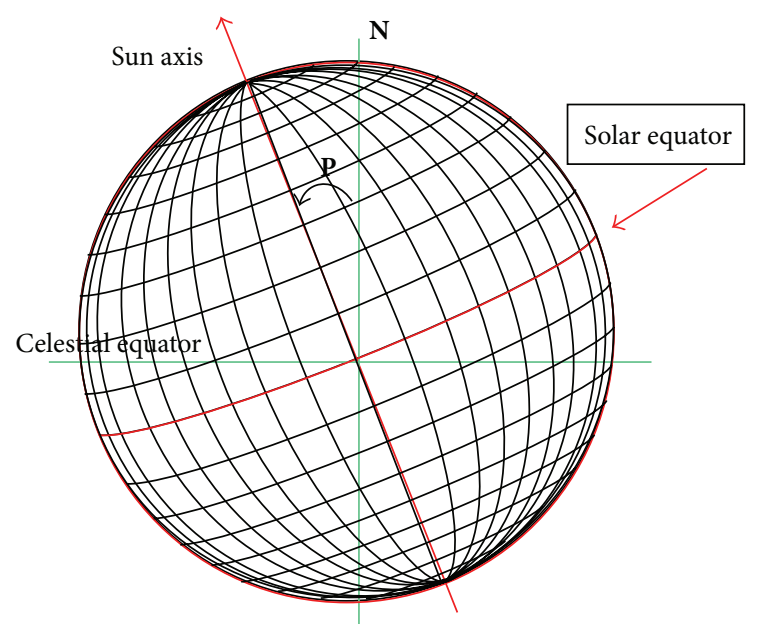

FIGURE 1: Heliographic coordinates where $L_{0}$ is the longitude of the heliographic center, $B_{0}$ is the latitude of the heliographic center, and $P$ is azimuth of the sun's axis.

\section{Methods}

\subsection{Method I}

3.1.1. Determining the Sun's Rotation Axis. We observed the direction of the movement of the same sunspots to judge the rotation direction of the sun. The movement of sunspots is mainly caused by the rotation of the sun, and their trajectory should be parallel and perpendicular to the sun's rotation axis which is the solar equator. The sun is a sphere projection, so the image of a large sunspot movement trajectory is presented by a curve. Therefore, the orientation of the sun from east to west cannot be obtained by any tracking all the lines of sunspots. We use Math PS to trace the arc of the sunspots. The two connections where the solar disk edge and trajectory arc intersect indicate the east and the west of the sun.

3.1.2. Azimuth Angle $P$ between the Sun and the Earth's Rotation Axis of Rotation Axis. Because the rotation axis (north-south) must be perpendicular to the latitude line (east-west), the angle between the east-west line of Sun and the east-west line of Earth in the image can be substitute for $P$.

3.1.3. Production of Solar Latitude and Longitude Lines. See Figure 1.

\subsubsection{Sunspots in Heliographic Position Measurements}

(1) Calibration Values $B_{0}$. After selecting a suitable view of the sun's surface from the astronomical yearbook, we overlapped the images with a projection surface map of the solar panel. By moving the projection board back and forth the latitude and longitude lines can coincide with each other (Figure 2). From $B_{0}=0^{\circ}- \pm 7^{\circ}, \pm 1^{\circ}$, there are a total of eight intervals.

(2) $P$ Values of Calibration. To locate the regularity of the sun's rotation axis in different seasons, the sun's latitude and 


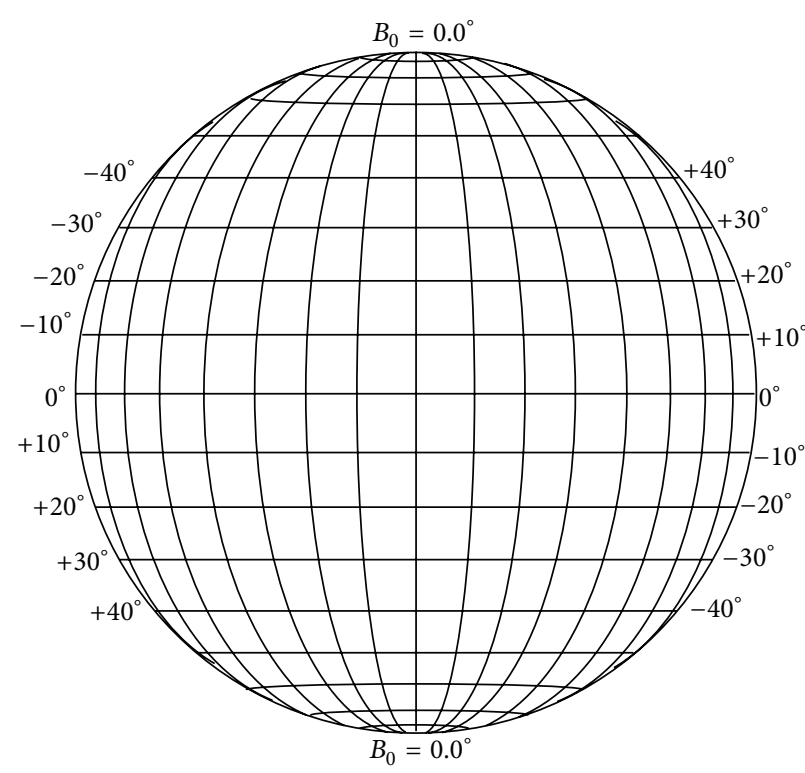

(a)

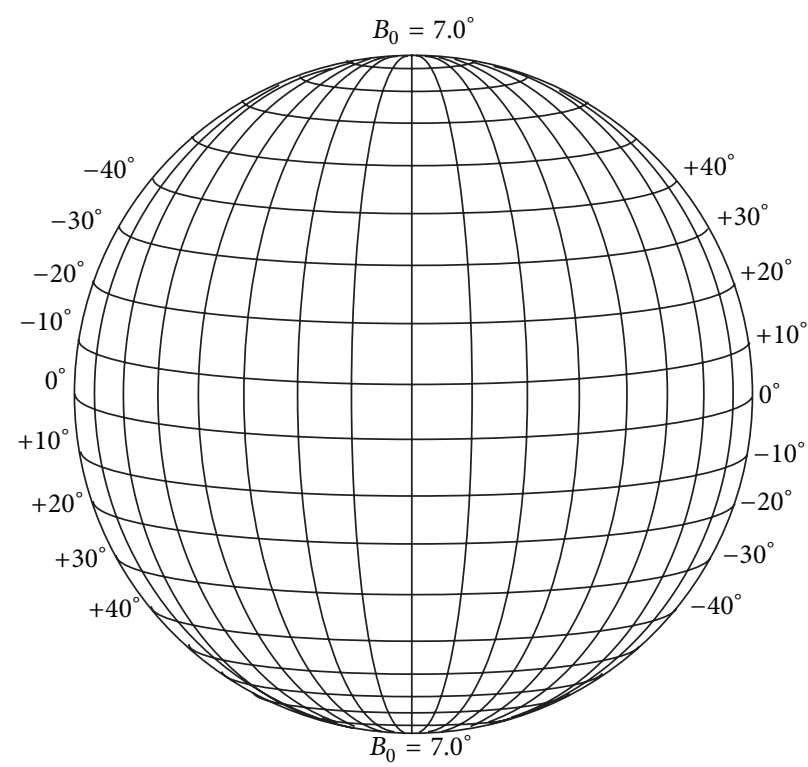

(b)

Figure 2: (a) The center of the solar disk is 0-degree latitude. (b) The center of the solar disk is 7-degree latitude.

longitude lines can be rotated to fit with its rotation axis. The angle between the rotation axes of the Sun and the Earth is not fixed and changes from $0^{\circ}$ to $26^{\circ}$; it will have an east or west bias of 0 to 26 degrees. In early January and early July, the angle is 0 degrees, while the angle in April and October is approximately 26.27 degrees.

3.1.5. Calculating the Solar Rotation Period. We use graphic software, Photo Impact, to measure the same sunspot's moving angle interval $(\Delta \theta)$ over a certain period of time. We then divide 360 degrees by the angle. The known sidereal period of the Earth revolving is 365.2564 days. Assuming that the solar sidereal rotation period is $T_{0}$ and the rendezvous period of the sunspot observation from Earth is $T_{s}$, then we have the following formula:

$$
\frac{360}{T_{0}}-\frac{360}{T_{s}}=\frac{360}{365.2564} .
$$

Thus, $T_{0}=365.2564 T_{s} /\left(365.2564+T_{s}\right)$.

3.2. Method II. By observing the track of the sunspots and the formula of the sidereal rotation period, the sun's rotation period can be calculated.

Step 1. Observe the sunspots' track and print the latitude and longitude lines on the slide; then post them on a computer screen to carefully compare them with each other (Figure 3 ). Another method is to implant the solar disk coordinate diagram in Photoshop, so that the two are superimposed.

Step 2. Identify the moving distance of the sunspots and then convert it to the movable angle $\Rightarrow$ angular displacement.

Step 3. Divided by the time, the angular velocity is obtained.

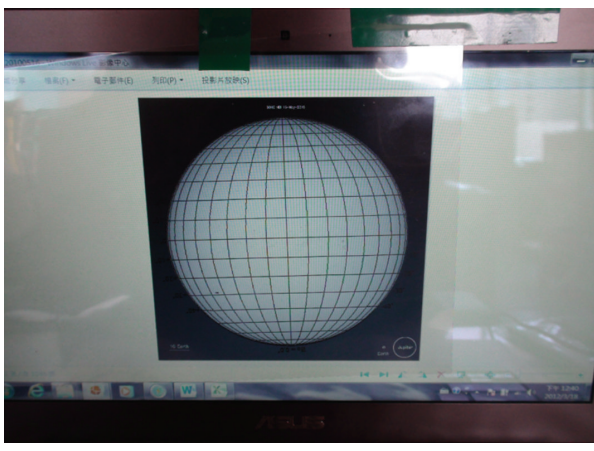

Figure 3: Posted slides on the computer screen for observation.

Step 4 . The 360/angular velocity = sunspot cycle $S$.

Step 5. Substitute $P=S \times E /(S+E)$, where $P$ is the sidereal period, $S$ is the sunspot cycle, and $E$ is the Earth to date = 365.2564 days.

3.3. Method III: Calculation of the Solar Rotation Period. For example, The heliolongitude $L$ of sunspot number 1642 in 0103-2013 is $252.16^{\circ}$. After 6 days, heliolongitude $L=252.34^{\circ}$ (Table 1).

Within 6 days, heliolongitude changed from $252.34^{\circ}$ to $252.16^{\circ}$, that is, $0.18^{\circ}$.

The sunspot spent 25.38 days rotating around $\left(360^{\circ}\right)$; that is, $360 / 25.38=14.184^{\circ} /$ day.

Within 6 days, the sunspot moved $14.184^{\circ} \times 6=85.104^{\circ}$.

Therefore, the sunspot changed $85.104^{\circ}+0.18^{\circ}=85.284^{\circ}$ within 6 days; that is, the sunspot changed $85.284^{\circ} / 6=14.214^{\circ}$ in one day.

As a result, the Sun's stellar period is $360 / 14.214=25.3271^{\circ}$. 
TABLE 1: Part of the observation record of 2013 sunspots (taking 6 days).

\begin{tabular}{cccccc}
\hline & A & B & C & D & E \\
& Date & Sunspot number & Heliolongitude $L$ & Heliographic latitude $B$ & Solar rotation period $P$ \\
\hline 1 & 20131203 & 1642 & 252.16 & -12.93 & - \\
2 & 20130109 & 1642 & 252.34 & -24.71 & - \\
3 & 20130131 & 1665 & 243.77 & 10.72 & 25.33 \\
4 & 20130206 & 1665 & 244.4 & 12.44 & - \\
5 & 20130212 & 1671 & 75.62 & 14.16 & 24.95 \\
6 & 20130218 & 1671 & 77.07 & 14.23 & - \\
7 & 20130312 & 1692 & 77.1 & 9.38 & 25.19 \\
8 & 20130318 & 1692 & 77.74 & 8.95 & 25 \\
\hline
\end{tabular}

Excel formula (take two decimals) $\mathrm{E} 2=360 /(((360 / 25.38) * 6)+(\mathrm{C} 2-\mathrm{C} 1)) / 6)=25.33$.

\section{Results and Discussion}

From Methods I to III, the sun's rotation period can be calculated. Among them, two of the most convenient methods are taking continuous moving track records of 3 days and 6 days to calculate the sun's rotation period. Although the results were not identical, the difference between them was small. As a result, the solar rotation period can be accurately estimated by these methods. From the observations of sunspots in January, July, and October of 2013, we could find that the rotation period of the sun was about 25 days (taking 3 days).

\section{Conclusion}

By observing the moving angular rate of sunspots on the solar disk and combining with sidereal rotation period expressions to calculate the rotation period of solar, the results show that we can not only estimate the rotation period of solar more conveniently and accurately, but also simulate the movement trajectory of sunspots further and estimate the change and the sloping angle of the sun's rotation axis relative to the Earth's rotation axis.

\section{Conflict of Interests}

The authors declare that there is no conflict of interests regarding the publication of this paper.

\section{References}

[1] H. W. Babcock, “The topology of the sun's magnetic field and the 22-year cycle," The Astrophysical Journal, vol. 133, no. 2, pp. 572-587, 1961.

[2] J. Javaraiah, "Long-term variations in the solar differential rotation," Solar Physics, vol. 212, no. 1, pp. 23-49, 2003.

[3] C. E. St. John, "The present condition of the problem of Solar Rotation," Publications of the Astronomical Society of the Pacific, vol. 30, no. 178, article 319, 1918.

[4] K. J. Li, W. Feng, X. J. Shi, J. L. Xie, P. X. Gao, and H. F. Liang, "Long-term variations of solar differential rotation and sunspot activity: revisited," Solar Physics, vol. 289, no. 3, pp. 759-768, 2014. 


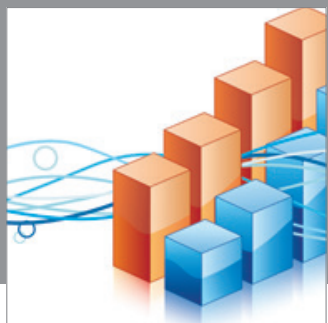

Advances in

Operations Research

mansans

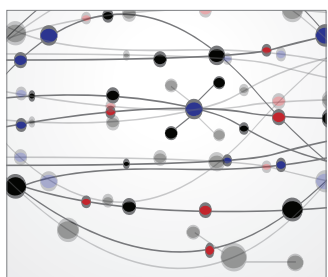

The Scientific World Journal
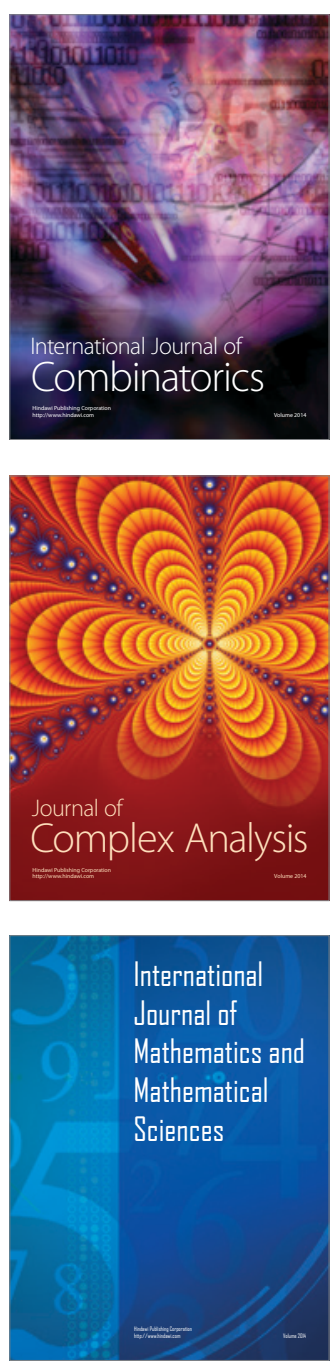
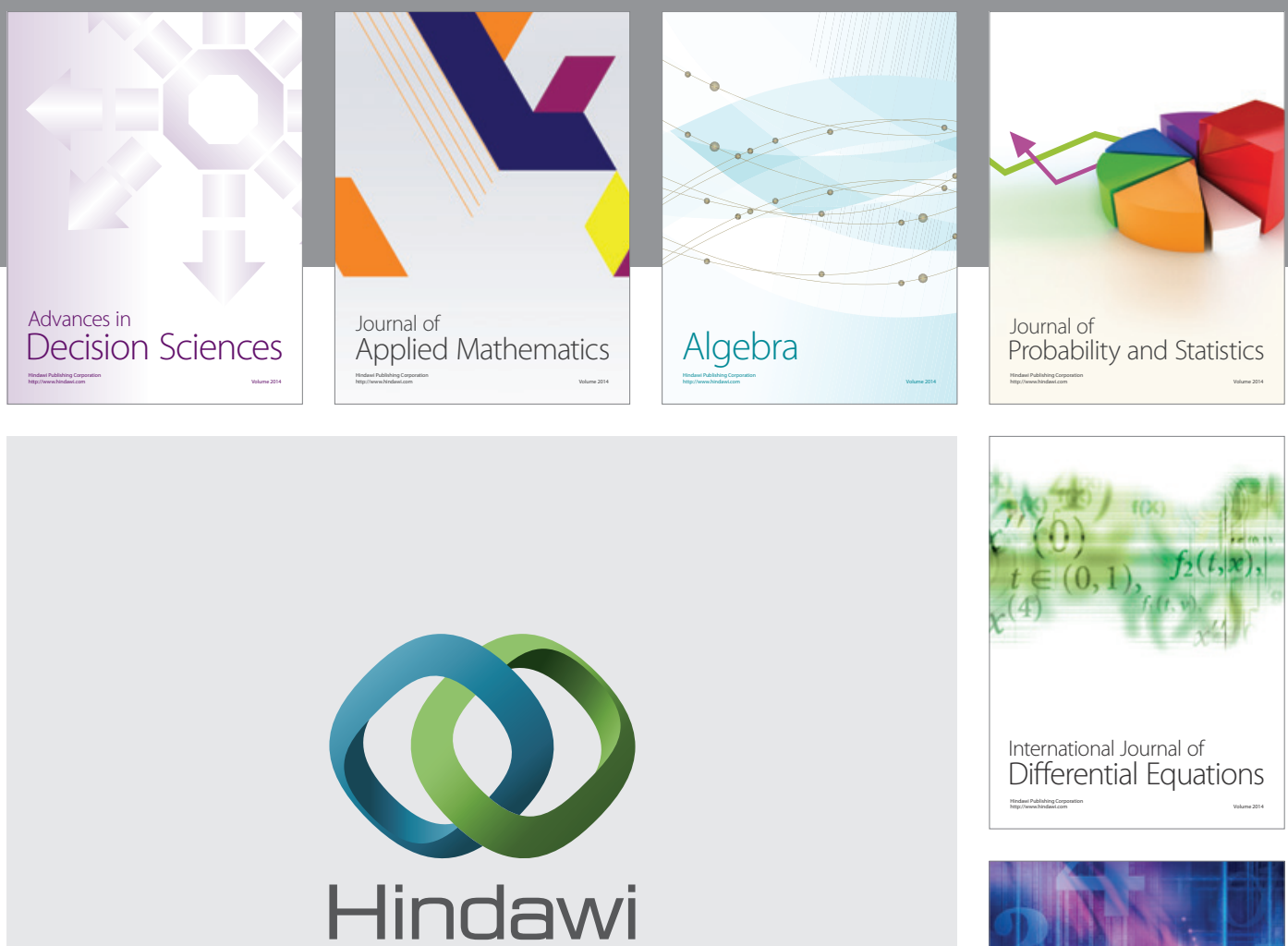

Submit your manuscripts at http://www.hindawi.com
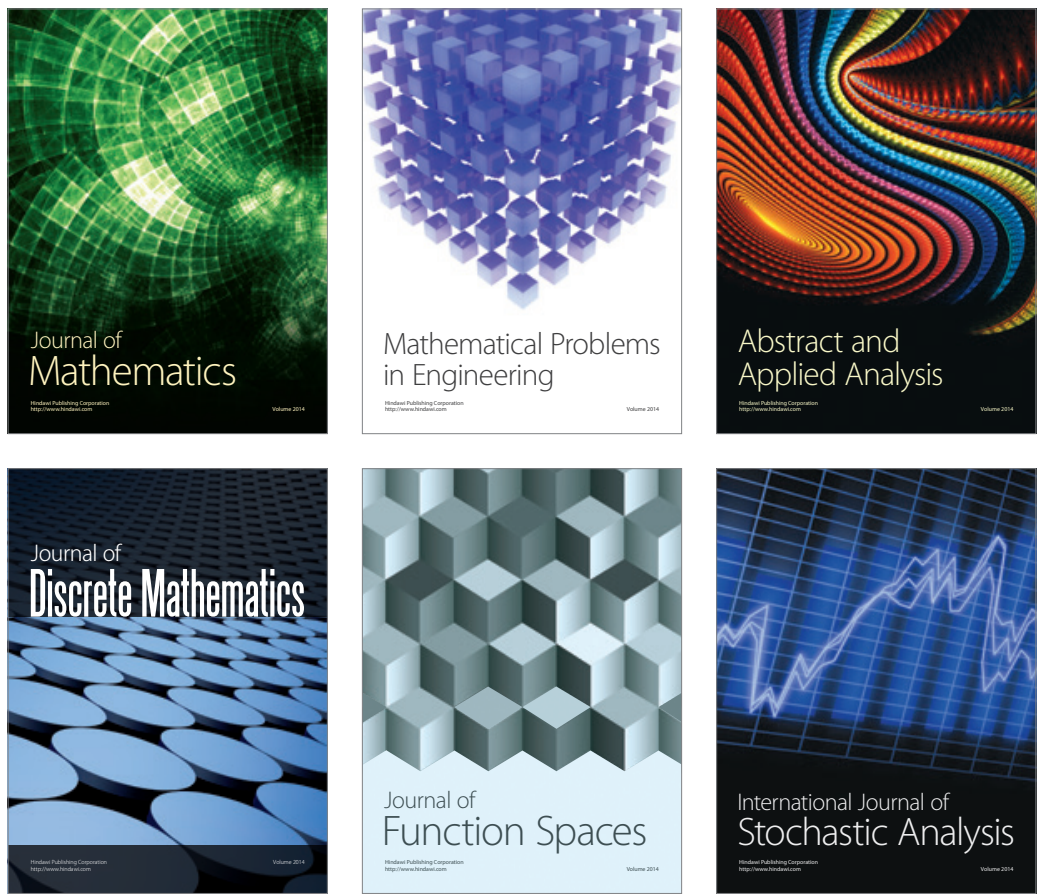

Journal of

Function Spaces

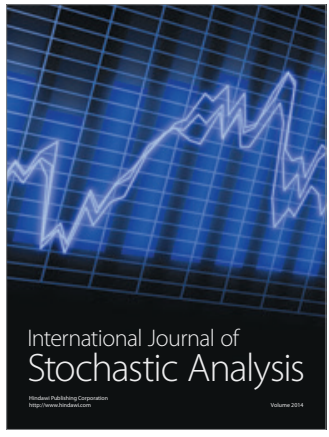

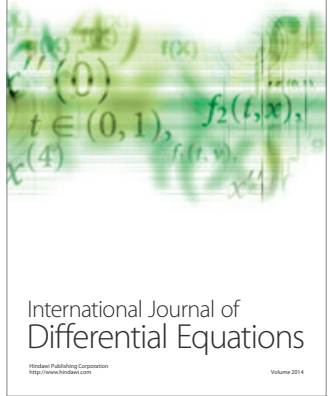
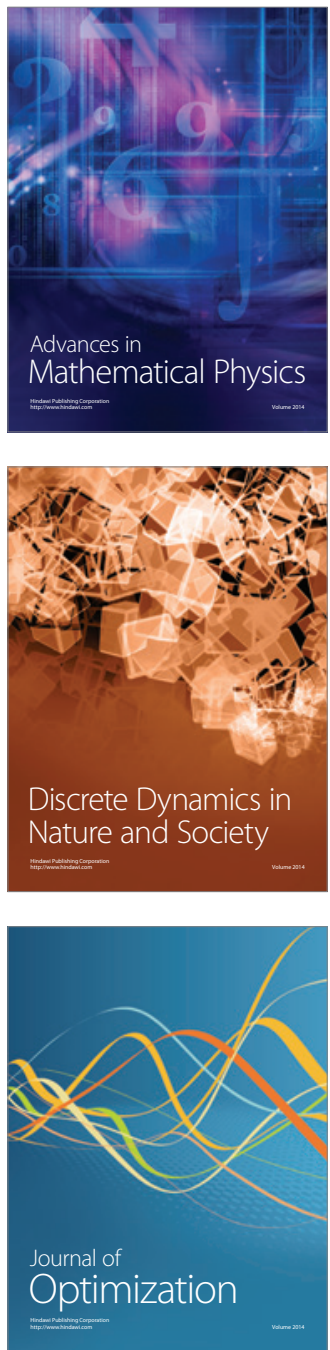